\title{
Estudio arqueomagnético de cerámicas del Holoceno elaboradas por sociedades indígenas del Uruguay
}

\author{
Juan Morales, Leda Sánchez-Betucci, Irina Capdepont, Avto Goguitchaichvili
}

\begin{abstract}
Juan Morales
jmorales@geofisica.unam.mx

Laboratorio Interinstitucional de Magnetismo Natural y Servicio Arqueomagnético Nacional, Instituto de Geofísica, UNAM, Unidad Michoacán, Campus Morelia. Antigua Carretera a Pátzcuaro No. 8701 Col. Ex-Hacienda de San José de la Huerta. 58190 Morelia, Michoacán México.
\end{abstract}

\section{Avto Goguitchaichvili}

Laboratorio Interinstitucional de Magnetismo Natural y Servicio Arqueomagnético Nacional, Instituto de Geofísica, UNAM, Unidad Michoacán, Campus Morelia. Antigua Carretera a Pátzcuaro No. 8701 Col. Ex-Hacienda de San José de la Huerta. 58190 Morelia, Michoacán México.

\section{Leda Sánchez-Betucci}

Facultad de Ciencias, Universidad de la República de Uruguay, Montevideo. Iguá 4225, Malvín Norte, 11400, Montevideo, Uruguay.

\section{Irina Capdepont}

CURE - UdelaR y Laboratorio de Estudios del Cuaternario (MEG/UNCIEP-IECA, Facultad de Ciencias) Iguá 4225, Piso 11, Ala Sur. 11400, Montevideo, Uruguay.

BOL. SOC. GEOL. MEX. 2019

VOL. 71 NO. 2

P. $481-495$

http://dx.doi.org/10.18268/BSGM2019v7 ln2a15

Manuscrito recibido: Enero 14, 2018.

\section{RESUMEN}

Las variaciones del campo geomagnético en escalas de tiempo arqueológico pueden obtenerse a partir del estudio de estructuras quemadas y cerámica arqueológica fechadas. Presentamos los primeros resultados de arqueointensidad obtenidos de cerámicas del Uruguay elaboradas por sociedades indígenas que ocuparon diversos espacios del territorio uruguayo. Los datos obtenidos de curvas termomagnéticas continuas y de adquisición de magnetización isotermal remanante indican que la titanomagnetita pobre en titanio es la responsable de la remanencia en estos fragmentos cerámicos. Quince fragmentos ( 100 especímenes $)$ de los veintidós analizados produjeron estimaciones confiables de intensidad. Las determinaciones de arqueointensidad se obtuvieron empleando el método de Thellier-Coe, mientras que los fechamientos correspondientes utilizando la herramienta de datación arqueo_dating. Las arqueointensidades obtenidas del total de las muestras analizadas varían entre $(23.3 \pm 1.4) \mu \mathrm{T}$ у $(42.9 \pm 2.4) \mu \mathrm{T}, \mathrm{y}$ corresponden a edades entre 1660 AEG y 1660 EG. Dichas edades son consistentes con las cronologías obtenidas por ${ }^{14} \mathrm{C}$ reportadas para los sitios arqueológicos de donde proviene la cerámica.

Palabras clave: Cerámicas indígenas de Uruguay, Holoceno, Arqueointensidad geomagnética, Fechamiento arqueomagnético.

\section{ABSTRACT}

The study of well-dated archeological potsherds and heated structures (i.e. kilns, floors, etc.) yields the opportunity for a better knowledge of the geomagnetic field variations at archeological timescales. We present the first archeointensity results obtained on Uruguayan ceramics prepared by indigenous societies on the coast of the Uruguay River. Titanomagnetite with low Ti content seem to be the responsible for the remanence in these ceramic fragments, as inferred from the results obtained from continuous thermomagnetic and isothermal remanent magnetization acquisitions curves. Fifteen ( 100 specimens) out of the twenty-two analyzed fragments produced reliable archeointensity estimates. Archeointensity determinations were obtained using the Thellier-Coe method, while the corresponding archeointensity dates by using the dating arqueo_dating tool. Obtained archeointensities from the total of the analyzed samples vary between $23.3 \pm 1.4 \mu T$ and 42.9 $\pm 2.4 \mu \mathrm{T}$, and correspond to ages between $1660 B C$ and $1660 A D$. These ages are consistent with ${ }^{14} C$ dates previously reported in the area.

Keywords: Indigenous societies of the Uruguay, Holocene, Geomagnetic Archeointensity, Archeomagnetic dating. 


\section{Introducción}

El material cerámico se considera como una de las tareas artesanales más antiguas que los seres humanos han realizado. Su estudio permite reconocer aspectos tecnológicos y productivos de las sociedades que lo manufacturó, utilizó y desechó, aportando datos sobre economía, alimentación, comercio, intercambio, movilidad, organización social y costumbres, entre otros (Shepard, 1954; Rice, 1987; Cobas y Prieto, 1998; Prieto, 2003).

Actualmente en Uruguay no se cuenta con evidencias contundentes que permitan relacionar los materiales cerámicos, adjudicados a distintos grupos étnicos, a un espacio y tiempo concreto. A pesar de que varios autores, desde los inicios de las investigaciones arqueológicas en Uruguay, han propuesto clasificaciones y modelos de ocupación prehistórica (Serrano 1933, 1936, 1972; Austral, 1977; Díaz y Fornaro, 1977; Hilbert, 1991), los límites cronológicos aún siguen siendo difusos. En este sentido, el presente trabajo busca contribuir con información que permita ajustar con mayor precisión la temporalidad de la cerámica del territorio uruguayo.

Las investigaciones de campo y laboratorio llevadas adelante por Capdepont (2013) han ubicado diversas zonas arqueológicas alfareras, situadas en distintas unidades del paisaje [terrenos elevados por acción natural (albardones) y antrópica (estructuras monticulares en tierra - cerritos), costas de río, estuario y océano] utilizadas como áreas formales de enterramientos y asentamientos. Las investigaciones ponen en evidencia que durante el Holoceno existieron las condiciones propicias y estratégicas para el establecimiento de diferentes grupos indígenas alfareros que practicaron la caza, recolección, pesca, horticultura y/o agricultura. Para estos grupos se postula una alta conectividad social y cultural (Capdepont, 2013). Desde el punto de vista del arqueomagnetismo, Sudamérica puede considerarse aún como tierra virgen. No obstante, se han realizado estudios en Perú (Shaw et al., 1996) y Ecuador
(Bowles et al., 2002) pero sin mediciones sistemáticas de arqueointensidad. La principal limitación de este tipo de estudios es que la mayoría del material arqueológico disponible para estudios arqueomagnéticos es material no orientado (i.e. piezas utilitarias como vasijas o aquellas empleadas en rituales ceremoniales), lo cual limita el número de determinaciones de arqueodirecciones (declinación e inclinación magnética) del campo geomagnético. En contraste, un estudio de arqueointensidad absoluta tiene la gran ventaja de que no requiere de material orientado.

Recientemente Hartmann et al. (2009) reportaron los primeros resultados de la arqueointensidad del noreste de Brasil, obtenidos de 14 grupos de fragmentos de ladrillo arquitectónico muestreados en la ciudad de Salvador, estado de Bahía. Sin embargo, el estudio está restringido a un intervalo de tiempo muy corto, entre mediados del siglo XVI y principios del siglo XIX. Asimismo, Goguitchaichvili et al. (2012) llevaron a cabo una investigación de arqueointensidad en algunos fragmentos cerámicos seleccionados de los humedales del Paraná inferior (región Pampeana, Argentina) con el fin de construir la primera curva maestra de arqueointensidad para Sudamérica. No obstante, el estudio está restringido a un intervalo de tiempo muy reciente, entre $410 \mathrm{AD}$ y $1285 \mathrm{AD}$.

Debido a la escasa aplicación de métodos arqueomagnéticos en la reconstrucción cronológica relacionada con las etapas iniciales de ocupación humana con alfarería en Uruguay, este trabajo aporta datos cronológicos confiables, sustentados con evidencias arqueológicas o dataciones de contextos cerámicos. Se analizaron 15 fragmentos cerámicos recuperados de diversos sitios arqueológicos ubicados en cinco departamentos de Uruguay. Diez de estos fragmentos provienen de contextos arqueológicos datados por ${ }^{14} \mathrm{C}$ (Sitio La Esmeralda, Cráneo Marcado, Guardia del Monte, García Ricci y Yale 27) y tres fragmentos presentan cronologías por TL (Tiestos del Sitio Guayacas). Las cronologías arqueomagnéticas realizadas sobre los propios 
fragmentos cerámicos, obtenidas en esta investigación, concuerdan con los datos cronológicos previos de los contextos de hallazgo del material. Ello estaría confirmando la utilidad de esta metodología como herramienta alternativa para el fechamiento. Para los 3 fragmentos restantes (Sitio Isla de Arriba, Isla Mellizas y costa de Canelones), carentes de cronologías previas, se estimaron los intervalos de edad más probables.

\section{2. Área de estudio}

El área de estudio comprende diferentes sitios arqueológicos localizados en cinco departamentos del Uruguay (Figura 1). Dos de estos sitios, Guayacas (en un tope de lomada) e Isla mellizas (en el río Uruguay) se ubican en el departamento de Paysandú. Este departamento se encuentra en el litoral oriental del río Uruguay, al igual que el

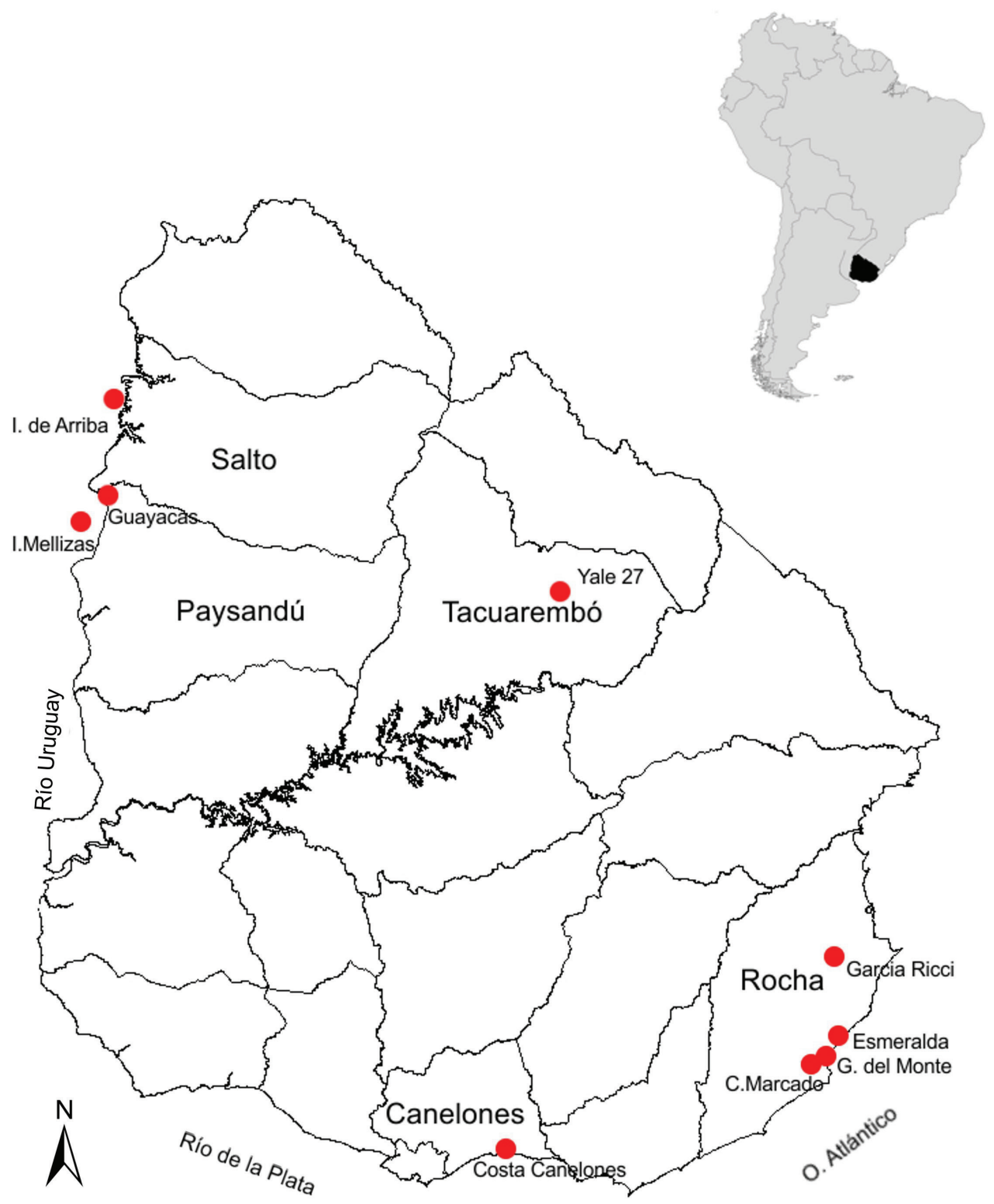


departamento de Salto, donde se ubica el sitio Isla de Arriba. En el departamento de Tacuarembó, ubicado en el centro-norte del territorio, se encuentra el sitio Lemos, en donde se encuentra la estructura monticular Yale 27. Otros cuatro sitios se encuentran en el departamento de Rocha, ubicado al sur-este de Uruguay. Tres de estos sitios (Cráneo Marcado, Guardia del Monte y García Ricci) se caracterizan por la presencia de cerritos de indios y el otro (La Esmeralda) está conformado por acumulaciones de material biogénico (concheros) y materiales superficiales en los espacios interdunares. En el departamento de Canelones el material se encuentra en la costa de confluencia del Río de la Plata y el océano Atlántico.

\section{Materiales y métodos}

De los sitios mencionados (=9) se analizaron 16 fragmentos de cerámica. Cuatro de los fragmentos muestreados son del departamento de Paysandú (tiestos 7, 12, Gy0633 y Gy04042), uno de Salto (tiesto 5611), tres de Tacuarembó (tiestos 47, 9 y 29), siete de Rocha (tiestos 8, 11, GM01, CM03, 5, CO08 y CO13) y uno de Canelones (tiesto 10). Estos fragmentos fueron muestreados mediante su seccionamiento en cuadros de $\sim 1 \mathrm{~cm} \times 1 \mathrm{~cm}$, obteniendo entre 6 y 7 especímenes de cada uno. Los especímenes así obtenidos fueron prensados en pastillas cilíndricas de sal con dimensiones similares a las de las muestras paleomagnéticas estándares para facilitar su tratamiento (Figura 2).
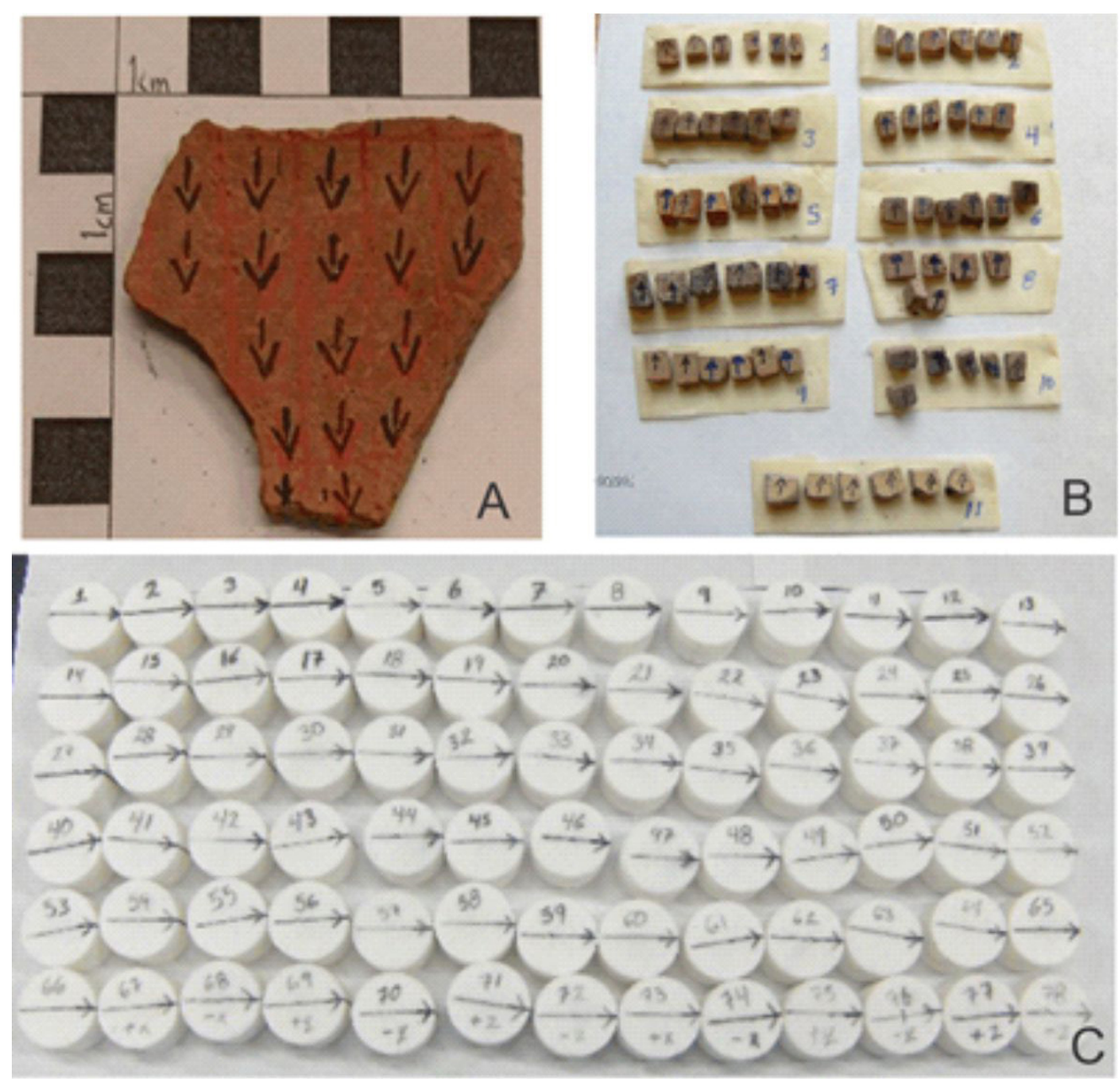

Figura 2 (a) Muestra representativa de cerámica, marcada para seccionarse; (b) cerámica seccionada en cuadros de $\sim 1 \mathrm{~cm}^{2}$; (c) pastillas de sal conteniendo un fragmento cerámico. 
La determinación de propiedades magnéticas se realizó en muestra pulverizada $(300 \mathrm{mg})$, la cual fue obtenida al pulverizar los pequeños fragmentos resultantes del proceso de seccionado descrito anteriormente, mediante el empleo de una balanza termomagnética (AVFTB), lo que generó las curvas siguientes: adquisición de IRM, campo reverso, histéresis magnética y curva termomagnética. El análisis de las curvas obtenidas se llevó al cabo por medio del software RockMagAnalyzer 1.0 (Leonhardt, 2006).

La determinación de arqueointensidades se realizó por medio de experimentos tipo Thellier-Coe

(a)

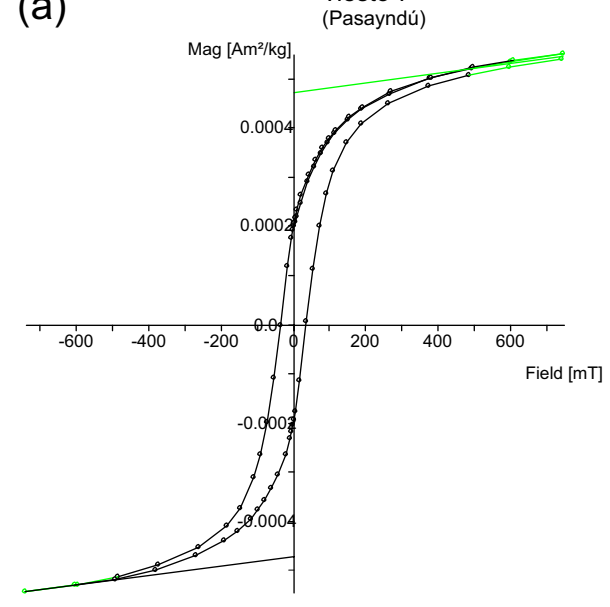

(c)

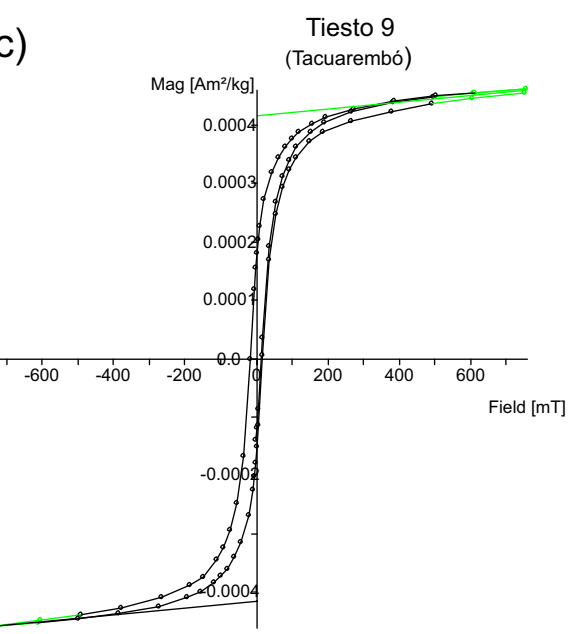

(Thellier y Thellier, 1959; Coe, 1967), utilizando para ello un horno ASC Scientific TD48-SC. Se realizaron diez pasos dobles de temperatura que fueron distribuidos a través del intervalo total de temperatura empleado $\left(25^{\circ} \mathrm{C}-540^{\circ} \mathrm{C}\right)$, con una reproducibilidad de $\pm 2^{\circ} \mathrm{C}$ entre dos calentamientos a la misma temperatura. La intensidad de campo de laboratorio se estableció en $45 \pm 0.5 \mu \mathrm{T}$. Se añadieron al procedimiento de laboratorio las pruebas conocidas como $p$ TRM check's cada tercer paso de temperatura, así como las conocidas como pTRM Tail checks (Riisager y Riisager, 2001) a 2 temperaturas intermedias $\left(350\right.$ y $\left.450{ }^{\circ} \mathrm{C}\right)$. (b)

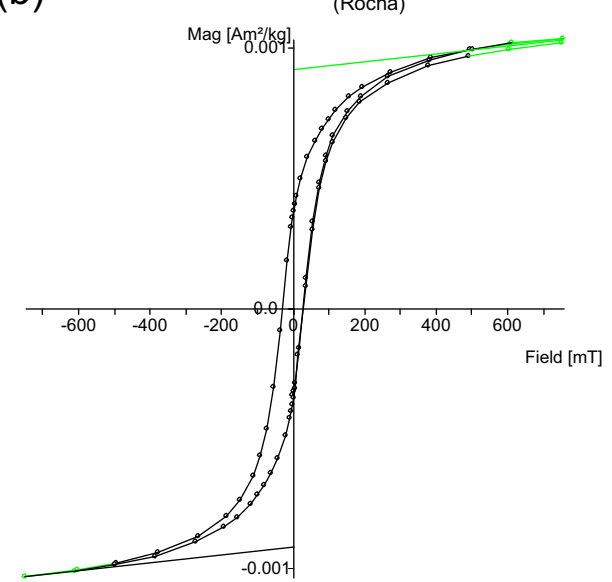

(d)

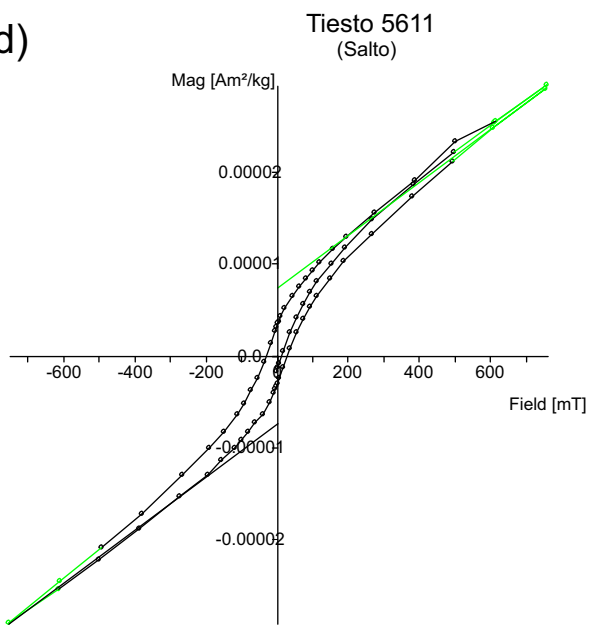

Figura 3 Ejemplos representativos de curvas de histéresis magnética de los fragmentos cerámicos analizados. (a) - (c) Curvas pertenecientes al tipo pot-bellied. (d) Curva perteneciente al tipo wasp-waisted. 
(a)

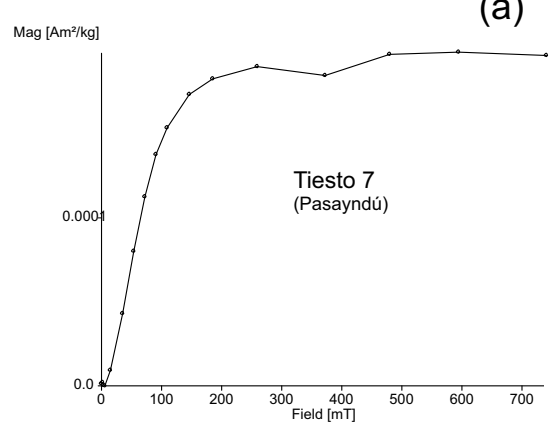

(c)

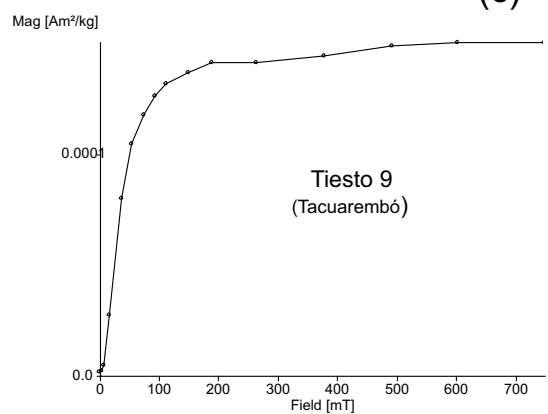

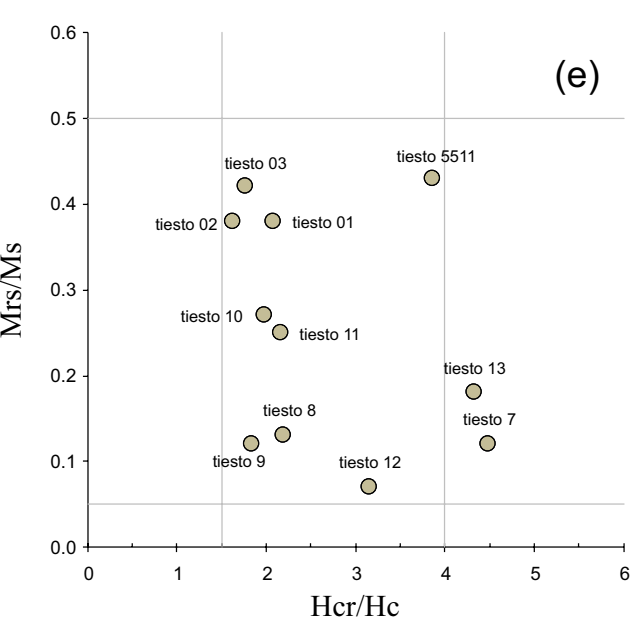

(e)

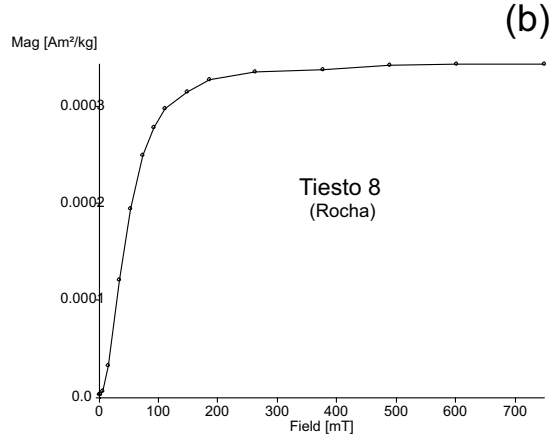

Figura 4 Ejemplos representativos de curvas de adquisición de IRM de los fragmentos cerámicos analizados. (a) - (c). La saturación se alcanza entre los 200 y $300 \mathrm{mT}$. (d) La saturación se alcanza hasta los $600 \mathrm{mT}$, sugiriendo la posible presencia de hematita. (e) Diagrama de Day en el que se observa que la mayoría de las muestras grafican en la región conocida como PSD.

El análisis de las determinaciones se llevó al cabo por medio del software ThellierTool 4.11 (Leonhardt et al., 2004).

La dependencia de la intensidad de la magnetización con el ritmo de enfriamiento fue investigada siguiendo un procedimiento modificado al descrito por Chauvin et al., 2000 (véase Morales et al., 2009), con un tiempo de enfriamiento lento de $6 \mathrm{~h}$ entre los $540^{\circ} \mathrm{C}$ y $20^{\circ} \mathrm{C}$.

Existen diferentes maneras para realizar las correcciones por anisotropía de la TRM (ATRM) como las propuestas por McCabe et al. (1985), Selkin et al. (2000), Chauvin et al. (2000), entre otros, que generalmente son procesos largos. Alternativamente, en esta investigación los especímenes de una misma muestra se prensaron (en pastillas de sal) colocados en seis posiciones diferentes a fin de disminuir el sesgo relacionado a los efectos de la ATRM, como se ha comprobado experimentalmente con anterioridad (Morales et al., 2007, 2011, 2012).

La datación relativa de piezas cerámicas (arqueológicas) se realiza por contexto o estilo cerámico, mientras que la datación absoluta de la mismas se efectúa mediante la técnica tradicional del radiocarbono $\left({ }^{14} \mathrm{C}\right)$ y, más recientemente, por medio de 
la termoluminiscencia (TL). Para la primera de éstas se requiere, necesariamente, la disponibilidad de materia orgánica certeramente asociada al objeto o contexto a datar. No obstante, se data el material orgánico, no la pieza propiamente. En el caso de la TL, una estimación certera de la paleo-dosis del sitio es igualmente requerida.

Alternativamente, la comparación de la intensidad del campo magnético antiguo $\left(\mathrm{H}_{\text {ant }}\right)$, grabada en las piezas cerámicas al momento de su cocción o última exposición al fuego, contra curvas de variación paleosecular (VPS) disponibles para los últimos milenios, permite determinar la edad

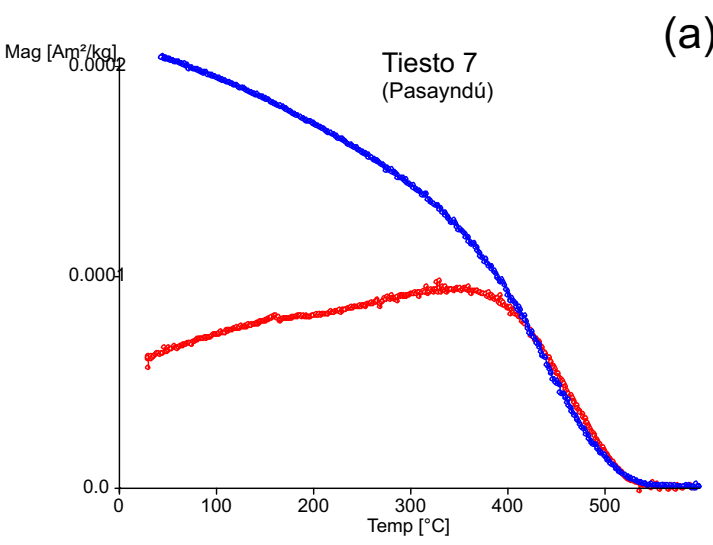

(c)

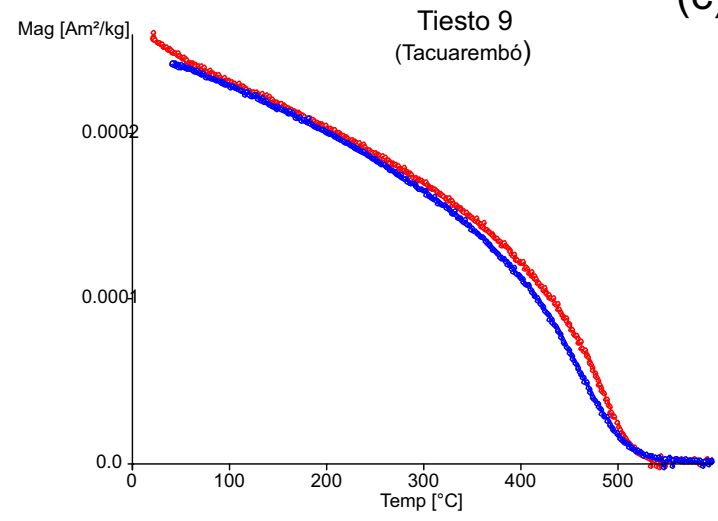

más probable de una pieza cerámica. En el caso de estructuras arcillosas in-situ (fogones, pisos y muros quemados) es posible además determinar la dirección antigua (declinación e inclinación) del $\mathrm{H}_{\text {ant }}$, y hacer la comparación de estos parámetros contra las curvas VPS correspondientes, obteniendo de esta forma el intervalo de edad más probable a partir de la probabilidad combinada de los tres parámetros.

En este estudio, el fechamiento arqueomagnético se realizó tras la obtención de las correspondientes arqueointensidades de las diferentes muestras; los resultados fueron analizados con la herramienta
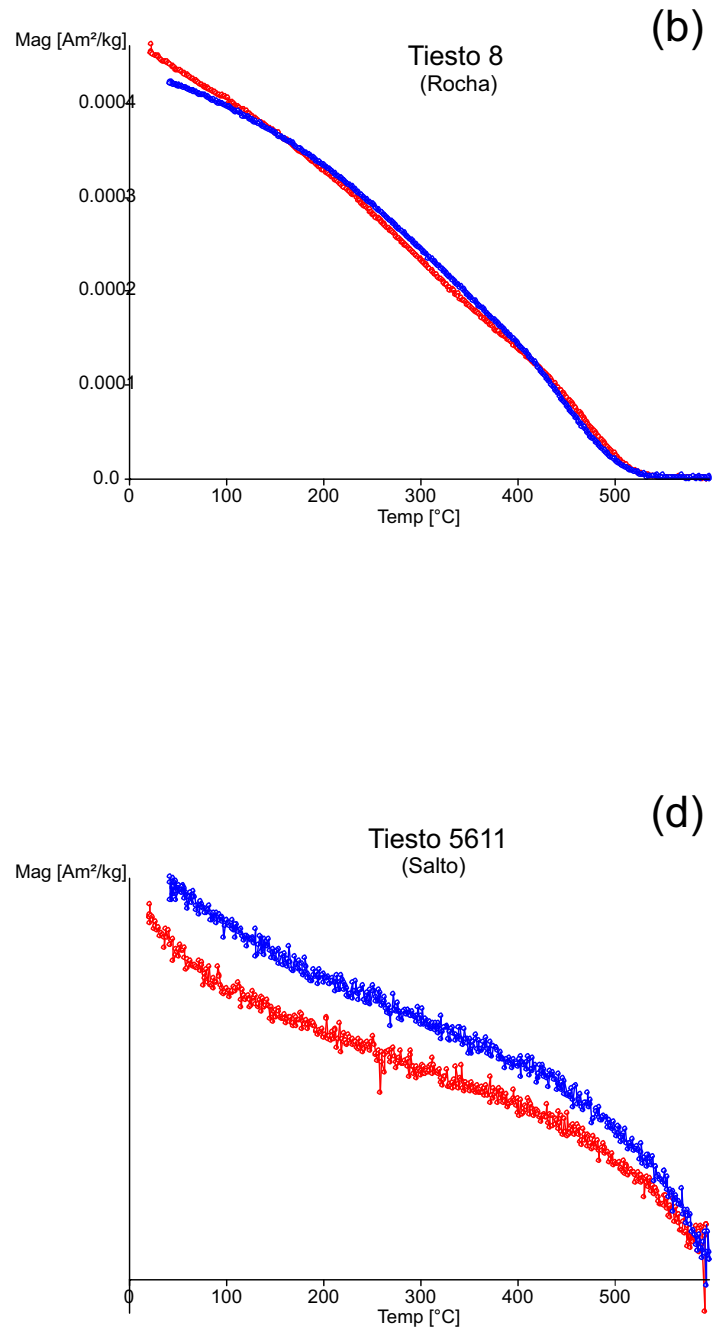
de datación arqueomagnética archaeo_dating, implementada en Matlab por Pavón-Carrasco et al. (2011).

\section{Resultados}

La forma de las curvas de histéresis es predominantemente del tipo pot-bellied (Figura 3a a 3c), con diferentecontribución paramagnética.Noobstante, la curva correspondiente al tiesto 5611 pertenece al tipo wasp-waisted (Figura 3d). La coercitividad $\left(\mathrm{B}_{\mathrm{c}}\right.$ ) varía entre los 4.5 y $40.0 \mathrm{mT}$, mientras que la coercituvidad de remanencia $\left(\mathrm{B}_{\mathrm{cr}}\right)$ entre los 11.3 y $154.6 \mathrm{mT}$. Por su parte, la magnetización de saturación $\left(\mathrm{M}_{\mathrm{s}}\right)$ varía entre $7.0 \times 10^{-6}$ y $9.7 \times$ $10^{-4} \mathrm{Am} / \mathrm{kg}$, mientras que la magnetización de saturación de remanencia $\left(\mathrm{M}_{\mathrm{rs}}\right)$ entre $3.0 \times 10^{-6}$ y $3.5 \times 10^{-4} \mathrm{Am} / \mathrm{kg}$. Todas las muestras alcanzan la saturación en campos alrededor de los 200-300 mT (Figura 4a a 4c), con excepción de la muestra tiesto 5611 , que lo hace hasta $\sim \operatorname{los} 600 \mathrm{mT}$ (Figura

Tabla 1. Resultados de arqueointensidad de tiestos fechados. Descripción de las diferentes columnas. Muestra: nombre del tiesto; Labname: nombre de laboratorio; Al: arqueointensidad; $\sigma$ : desviación estándar; Tmin/Tmax: temperatura mínima (máxima) utilizada para el ajuste por mínimos cuadrados; $\mathrm{N}$ : número de puntos utilizados para el ajuste; m: pendiente del mejor ajuste por mínimos cuadrados; ß: $\sigma / \mathrm{m} ; \mathbf{f}, \mathrm{g}$ y q: parámetros de calidad, definidos por Coe et al. 1978; MAD anchored: Desviación angular máxima anclada al origen; $\alpha$ : diferencia angular entre la mejor solución anclada y no anclada; Clase: (A, B o C); $\delta$ CK: error relativo de la verificación.

\begin{tabular}{|c|c|c|c|c|c|c|c|c|c|c|c|c|c|c|}
\hline Muestra & Labname & $\begin{array}{c}\text { AI } \\
{[\mu \mathrm{T}]}\end{array}$ & $\begin{array}{c}\sigma \\
{[\mu \mathrm{T}]}\end{array}$ & $\begin{array}{c}\operatorname{Tmin} / \mathrm{Tmax} \\
{\left[{ }^{\circ} \mathrm{C}\right]}\end{array}$ & $\mathbf{N}$ & m & $\beta$ & $f$ & $g$ & $q$ & $\begin{array}{c}\text { MAD } \\
\text { anchored }\end{array}$ & $\alpha$ & Clase & $\delta \mathrm{CK}$ \\
\hline Tiesto 7 & 90S1029A & 38.16 & 2.45 & $20-400$ & 5 & -1.27 & 0.06 & 0.55 & 0.57 & 4.9 & 5.2 & 8.6 & B & 4.3 \\
\hline Tiesto 7 & $90 \mathrm{~S} 1030 \mathrm{~A}$ & 46.34 & 5.04 & $20-400$ & 5 & -1.54 & 0.11 & 0.62 & 0.56 & 3.2 & 5.2 & 7.5 & B & 4 \\
\hline Tiesto 7 & $90 \mathrm{~S} 1031 \mathrm{~A}$ & 35.72 & 4.97 & $20-500$ & 8 & -1.19 & 0.14 & 0.73 & 0.77 & 4 & 4.8 & 7.1 & B & 4.2 \\
\hline Tiesto 7 & $90 \mathrm{~S} 1032 \mathrm{~A}$ & $\mathrm{~N} / \mathrm{R}$ & & & 0 & & & & & & & & & \\
\hline Tiesto 7 & $90 \mathrm{~S} 1033 \mathrm{~A}$ & $\mathrm{~N} / \mathrm{R}$ & & & 0 & & & & & & & & & \\
\hline Tiesto 7 & $90 \mathrm{~S} 1034 \mathrm{~A}$ & $\mathrm{~N} / \mathrm{R}$ & & & 0 & & & & & & & & & \\
\hline \multirow[t]{3}{*}{ Tiesto 7} & $90 \mathrm{~S} 1035 \mathrm{~A}$ & $\mathrm{~N} / \mathrm{R}$ & & & 0 & & & & & & & & & \\
\hline & prom $=$ & 40.1 & & & & & & & & & & & & \\
\hline & dst $=$ & 5.6 & & & & & & & & & & & & \\
\hline Tiesto 8 & $90 \mathrm{~S} 1036 \mathrm{~A}$ & $\mathrm{~N} / \mathrm{R}$ & & & 0 & & & & & & & & & \\
\hline Tiesto 8 & $90 \mathrm{~S} 1037 \mathrm{~A}$ & 32.86 & 1.61 & $20-560$ & 10 & -1.1 & 0.05 & 0.96 & 0.83 & 16.2 & 9.3 & 6.7 & $\mathrm{C}$ & 8.5 \\
\hline Tiesto 8 & 90S1038A & 32.66 & 1.29 & $20-560$ & 11 & -1.09 & 0.04 & 0.99 & 0.88 & 22.1 & 12.6 & 7.5 & B & 5.4 \\
\hline Tiesto 8 & 90S1039A & 36.31 & 1.54 & $20-560$ & 11 & -1.21 & 0.04 & 0.97 & 0.87 & 19.7 & 7.5 & 5.3 & B & 6.1 \\
\hline Tiesto 8 & $90 \mathrm{~S} 1040 \mathrm{~A}$ & 26.89 & 1.83 & $300-500$ & 6 & -0.9 & 0.07 & 0.5 & 0.76 & 5.6 & 2.7 & 2.6 & A & 4.8 \\
\hline Tiesto 8 & $90 \mathrm{~S} 1041 \mathrm{~A}$ & 31.42 & 1.44 & $20-560$ & 11 & -1.05 & 0.05 & 0.96 & 0.88 & 18.5 & 8.7 & 7.3 & B & 6.2 \\
\hline \multirow[t]{3}{*}{ Tiesto 8} & $90 \mathrm{~S} 1042 \mathrm{~A}$ & 33.29 & 1.56 & $20-560$ & 10 & -1.11 & 0.05 & 0.99 & 0.86 & 18.1 & 8.6 & 4.2 & B & 6.1 \\
\hline & prom $=$ & 32.2 & & & & & & & & & & & & \\
\hline & dst $=$ & 3.1 & & & & & & & & & & & & \\
\hline Tiesto 9 & $90 \mathrm{~S} 1234 \mathrm{~A}$ & 38.16 & 2.45 & $20-400$ & 5 & -1.27 & 0.06 & 0.55 & 0.57 & 4.9 & 5.2 & 8.6 & B & 4.3 \\
\hline Tiesto 9 & $92 \mathrm{~S} 1235 \mathrm{~A}$ & 33.16 & 3.3 & $20-500$ & 8 & -1.11 & 0.1 & 0.81 & 0.83 & 6.7 & 4.7 & 4.9 & B & 5.2 \\
\hline Tiesto 9 & $92 \mathrm{~S} 1236 \mathrm{~A}$ & 17.86 & 1.46 & $400-540$ & 5 & -0.6 & 0.08 & 0.59 & 0.73 & 5.3 & 3.1 & 3 & A & 4.7 \\
\hline Tiesto 9 & $92 \mathrm{~S} 1237 \mathrm{~A}$ & 29.72 & 2.4 & $20-500$ & 8 & -0.99 & 0.08 & 0.8 & 0.83 & 8.1 & 2.9 & 1.4 & A & 3.6 \\
\hline Tiesto 9 & $92 \mathrm{~S} 1238 \mathrm{~A}$ & 32.56 & 2.66 & $20-500$ & 8 & -1.09 & 0.08 & 0.84 & 0.82 & 8.5 & 2.8 & 4.5 & A & 2 \\
\hline \multirow[t]{3}{*}{ Tiesto 9} & $92 \mathrm{~S} 1239 \mathrm{~A}$ & 31.06 & 2.65 & $20-500$ & 8 & -1.04 & 0.09 & 0.89 & 0.83 & 8.6 & 6.2 & 6.1 & B & 2.8 \\
\hline & prom $=$ & 30.4 & & & & & & & & & & & & \\
\hline & dst $=$ & 6.8 & & & & & & & & & & & & \\
\hline
\end{tabular}


Tabla 2. Resultados de arqueointensidad de tiestos no fechados. Descripción de las diferentes columnas. Muestra: nombre del tiesto; Tmin/ Tmax: temperatura mínima (máxima) utilizada para el ajuste por mínimos cuadrados; $N$ : número de puntos utilizados para el ajuste; $\mathbf{f}, \mathrm{g}$ y q: parámetros de calidad, definidos por Coe et al. 1978; Al: arqueointensidad; $\sigma$ desviación estándar (Continúa en la siguiente página).

\begin{tabular}{|c|c|c|c|c|c|c|c|c|}
\hline $\begin{array}{c}\text { Departamento } \\
\text { Localidad }\end{array}$ & Muestra & $\begin{array}{c}\text { Tmin- } \\
\text { Tmax } \\
{\left[{ }^{\circ} \mathrm{C}\right]}\end{array}$ & $\mathbf{N}$ & $f$ & $g$ & $q$ & $\begin{array}{c}\text { AI } \\
{[\mu \mathrm{T}]}\end{array}$ & $\begin{array}{l} \pm 1 \sigma \\
{[\mu \mathrm{T}]}\end{array}$ \\
\hline Canelones & Tiesto 10 & $20-560$ & 10 & 0.82 & 0.66 & 3.3 & 18.01 & 2.95 \\
\hline \multirow[t]{5}{*}{ Costa } & & $20-500$ & 8 & 0.55 & 0.84 & 5.1 & 37.69 & 3.36 \\
\hline & & $20-515$ & 9 & 0.85 & 0.83 & 8.2 & 50.5 & 4.34 \\
\hline & & $20-500$ & 8 & 0.33 & 0.8 & 2.1 & 21.15 & 2.69 \\
\hline & & $20-500$ & 8 & 0.39 & 0.83 & 2.3 & 22.98 & 3.28 \\
\hline & & & & & & Prom $=$ & 44.1 & 9.1 \\
\hline Rocha & Tiesto 11 & $20-500$ & 8 & 0.67 & 0.84 & 9.7 & 36.03 & 2.08 \\
\hline \multirow{5}{*}{ Duna } & & $350-515$ & 6 & 0.44 & 0.78 & 7 & 38.04 & 1.84 \\
\hline & & $20-515$ & 9 & 0.69 & 0.85 & 14.1 & 44.65 & 1.87 \\
\hline & & $300-500$ & 6 & 0.44 & 0.78 & 9.4 & 37.81 & 1.38 \\
\hline & & $350-515$ & 6 & 0.45 & 0.77 & 6 & 34.26 & 2 \\
\hline & & & & & & Prom $=$ & 38.2 & 3.9 \\
\hline Pasayndú & Tiesto 12 & $20-450$ & 6 & 0.71 & 0.77 & 4.7 & 33.43 & 3.9 \\
\hline \multirow[t]{2}{*}{ Costa arroyo } & & $20-475$ & 7 & 0.84 & 0.79 & 7.6 & 34.5 & 2.99 \\
\hline & & & & & & Prom $=$ & 34 & 0.8 \\
\hline \multirow{6}{*}{$\begin{array}{l}\text { Rocha } \\
\text { Garcia Ricci } \\
008\end{array}$} & $\mathrm{CO} 08$ & $250-530$ & 8 & 0.618 & 0.814 & 3.15 & 21.42 & 3.42 \\
\hline & & $250-530$ & 9 & 0.596 & 0.826 & 4.91 & 24.71 & 2.48 \\
\hline & & $200-530$ & 10 & 0.673 & 0.851 & 5.55 & 23.54 & 2.43 \\
\hline & & $250-530$ & 9 & 0.608 & 0.84 & 5.57 & 24.53 & 2.25 \\
\hline & & $200-530$ & 10 & 0.676 & 0.85 & 6.31 & 22.23 & 2.03 \\
\hline & & & & & & Prom $=$ & 23.3 & 1.4 \\
\hline \multirow{6}{*}{$\begin{array}{l}\text { Rocha } \\
\text { Garcia Ricci } \\
013\end{array}$} & $\mathrm{CO} 13$ & $250-400$ & 4 & 0.456 & 0.628 & 1.54 & 42.53 & 7.92 \\
\hline & & $150-475$ & 8 & 0.748 & 0.809 & 6.98 & 36.36 & 3.15 \\
\hline & & $250-400$ & 4 & 0.341 & 0.664 & 2.44 & 45.14 & 4.19 \\
\hline & & $200-515$ & 9 & 0.65 & 0.832 & 9.84 & 35.19 & 1.94 \\
\hline & & $250-515$ & 7 & 0.677 & 0.795 & 6.34 & 36.59 & 3.11 \\
\hline & & & & & & Prom = & 39.2 & 4.4 \\
\hline Pasayndú & GY0H042 & $150-530$ & 11 & 0.846 & 0.866 & 10.59 & 31.86 & 2.21 \\
\hline \multirow[t]{6}{*}{ Guayacas } & & $200-515$ & 9 & 0.626 & 0.844 & 7.02 & 60.98 & 4.59 \\
\hline & & $150-500$ & 10 & 0.564 & 0.804 & 8.76 & 45.23 & 2.34 \\
\hline & & $150-500$ & 10 & 0.566 & 0.821 & 7.42 & 53.91 & 3.38 \\
\hline & & $150-500$ & 10 & 0.628 & 0.838 & 7.84 & 55.67 & 3.74 \\
\hline & & $150-500$ & 10 & 0.741 & 0.848 & 9.61 & 30.29 & 1.98 \\
\hline & & & & & & Prom = & 46.3 & 12.9 \\
\hline
\end{tabular}


Tabla 2. (Continuación) Resultados de arqueointensidad de tiestos no fechados. Descripción de las diferentes columnas. Muestra: nombre del tiesto; Tmin/Tmax: temperatura mínima (máxima) utilizada para el ajuste por mínimos cuadrados; N: número de puntos utilizados para el ajuste; f, g y q: parámetros de calidad, definidos por Coe et al 1978; Al: arqueointensidad; $\sigma$ desviación estándar.

\begin{tabular}{|c|c|c|c|c|c|c|c|c|}
\hline $\begin{array}{c}\text { Departamento } \\
\text { Localidad }\end{array}$ & Muestra & $\begin{array}{c}\text { Tmin- } \\
\text { Tmax } \\
{\left[{ }^{\circ} \mathrm{C}\right]}\end{array}$ & $\mathbf{N}$ & $f$ & $g$ & $q$ & $\begin{array}{c}\text { AI } \\
{[\mu T]}\end{array}$ & $\begin{array}{l} \pm 1 \sigma \\
{[\mu T]}\end{array}$ \\
\hline Rocha & $\begin{array}{c}\text { CRANEO } \\
\text { MARCADO } \\
03\end{array}$ & $150-540$ & 12 & 0.984 & 0.858 & 16.89 & 40.5 & 2.03 \\
\hline \multirow[t]{4}{*}{$\begin{array}{l}\text { Cerrito Laguna } \\
\text { de Castillos }\end{array}$} & & $150-475$ & 10 & 0.934 & 0.835 & 18.46 & 47.93 & 2.03 \\
\hline & & $150-540$ & 10 & 0.972 & 0.822 & 22.46 & 35.42 & 1.26 \\
\hline & & $150-500$ & 10 & 0.887 & 0.803 & 13.03 & 46.94 & 2.57 \\
\hline & & & & & & Prom $=$ & 42.7 & 5.9 \\
\hline Rocha & 5 & $150-515$ & 10 & 0.736 & 0.836 & 10.22 & 38.12 & 2.3 \\
\hline \multirow{6}{*}{ La Esmeralda } & & $150-515$ & 10 & 0.818 & 0.864 & 15.36 & 36.18 & 1.67 \\
\hline & & $150-450$ & 7 & 0.579 & 0.735 & 6.6 & 53.01 & 3.42 \\
\hline & & $150-515$ & 9 & 0.696 & 0.81 & 9.35 & 33.57 & 2.03 \\
\hline & & $150-500$ & 9 & 0.736 & 0.826 & 13.28 & 36.36 & 1.67 \\
\hline & & $150-515$ & 11 & 0.744 & 0.875 & 8.14 & 31.5 & 2.52 \\
\hline & & & & & & Prom $=$ & 38.1 & 7.7 \\
\hline Tacuarembó & 29 & $150-540$ & 10 & 0.898 & 0.858 & 10.72 & 27.54 & 1.98 \\
\hline \multirow[t]{2}{*}{ Yale } & & $150-475$ & 9 & 0.821 & 0.821 & 8.03 & 25.2 & 2.12 \\
\hline & & & & & & Prom $=$ & 26.4 & 1.7 \\
\hline Rocha & $\begin{array}{c}\text { CRANEO } \\
\text { MARCADO } \\
01\end{array}$ & $200-540$ & 9 & 0.855 & 0.844 & 34.93 & 43.56 & 0.9 \\
\hline \multirow[t]{6}{*}{$\begin{array}{l}\text { Cerrito Laguna } \\
\text { de Castillos }\end{array}$} & & $150-540$ & 10 & 0.88 & 0.861 & 19.58 & 45.36 & 1.76 \\
\hline & & $150-540$ & 11 & 0.857 & 0.865 & 27.43 & 44.96 & 1.22 \\
\hline & & $150-500$ & 9 & 0.78 & 0.808 & 22.67 & 43.7 & 1.22 \\
\hline & & $150-540$ & 10 & 0.912 & 0.856 & 34.82 & 40.14 & 0.9 \\
\hline & & $150-500$ & 8 & 0.743 & 0.763 & 20.09 & 39.87 & 1.13 \\
\hline & & & & & & Prom $=$ & 42.9 & 2.4 \\
\hline Paysandú & GY 0633 & $150-515$ & 10 & 0.516 & 0.862 & 8.42 & 42.57 & 2.25 \\
\hline \multirow[t]{6}{*}{ Guayacas } & & $150-540$ & 12 & 0.652 & 0.849 & 7.75 & 41.58 & 2.97 \\
\hline & & $150-540$ & 11 & 0.662 & 0.84 & 12.11 & 41.18 & 1.89 \\
\hline & & $150-500$ & 9 & 0.524 & 0.847 & 5.45 & 51.39 & 4.19 \\
\hline & & $150-500$ & 9 & 0.482 & 0.846 & 4.8 & 41.81 & 3.56 \\
\hline & & $200-515$ & 9 & 0.467 & 0.852 & 9.16 & 37.31 & 1.62 \\
\hline & & & & & & Prom $=$ & 42.6 & 4.7 \\
\hline
\end{tabular}


4d), sugiriendo en esta última la presencia de un mineral magnético de alta coercitividad, como la hematita. La localización de la mayoría de las muestras dentro del diagrama de Day se encuentra básicamente en la región de los dominios pseudo simples (PSD) (Figura 4e), con excepción de los tiestos 7 y 13. Las curvas termomagnéticas son bastante reversibles, excepto para el tiesto 7, presentando una fase ferrimagnética compatible con titanomagnetita pobre en Ti (Figura 5a a 5c). En caso del tiesto 5611 (Figura 5d), su temperatura de Curie (Tc) es mayor a los $600{ }^{\circ} \mathrm{C}$, lo que podría sugerir la presencia de hematita.

Los resultados de arqueointensidad obtenidos del tiesto 7 (con cronología por TL) y los obtenidos para los tiestos 8 y 9 (con cronología de contexto por ${ }^{14} \mathrm{C}$ ), corregidos por ritmo de enfriamiento, se presentan en la Tabla 1, junto con diferentes parámetros de calidad. De la misma forma, en la Tabla 2 se exponen los resultados correspondien-
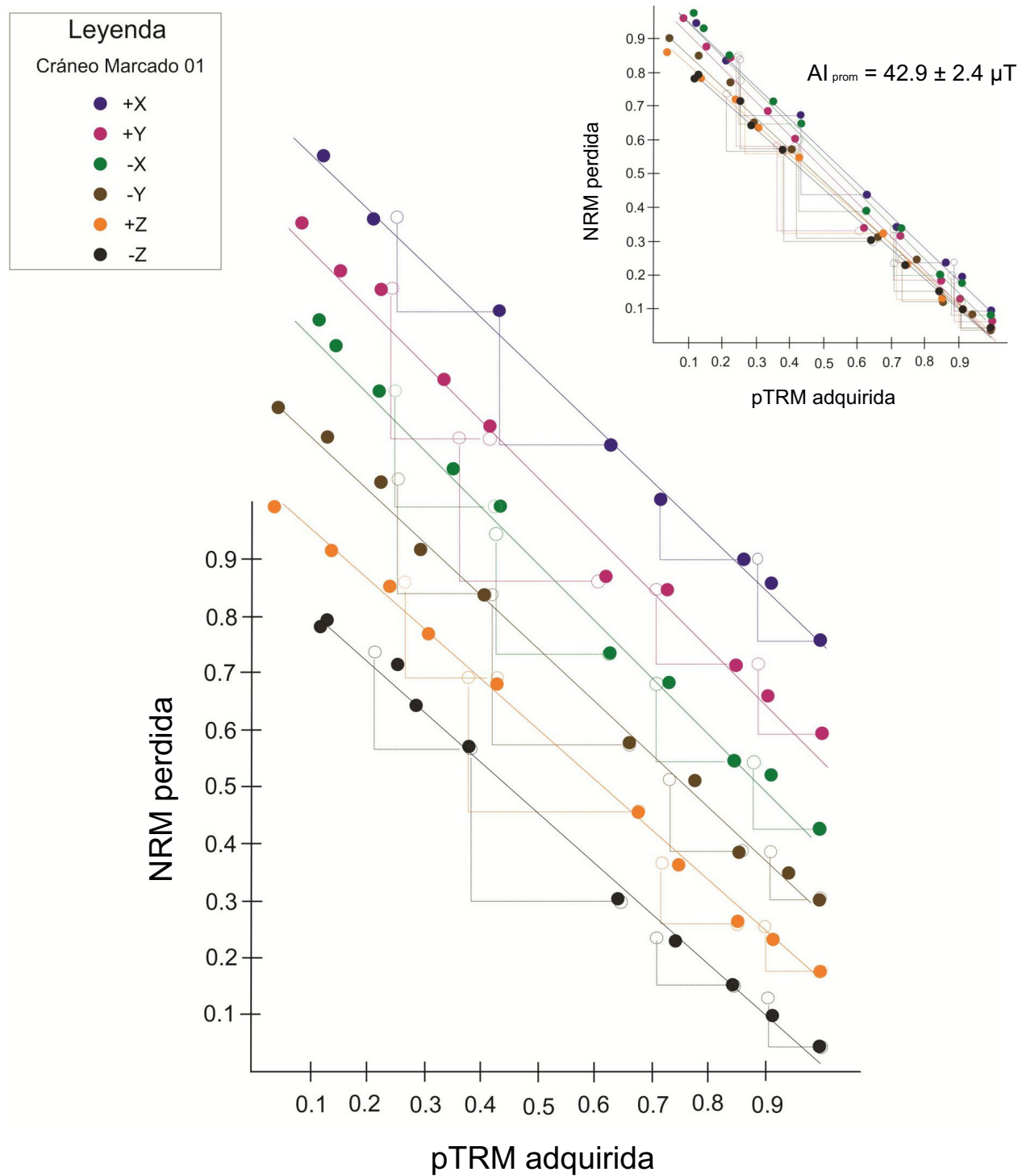

Figura 6 Resultados de arqueointensidad de los 6 especímenes obtenidos de una misma muestra (tiesto CM01). En la parte superior derecha se muestran en el mismo diagrama de Arai los valores normalizados para los seis especímenes. Las pruebas conocidas como PTRM check's se ilustran con las líneas punteadas con terminación en círculo vacío. En la parte central se grafican desplazadas para resaltar su paralelismo y, por tanto, la similitud de arqueointensidades obtenidas. 
tes a los otros 11 fragmentos estudiados, dos de los cuales carecen de datos cronológicos (tiesto 10 y 12). En la Figura 6 se observan los resultados de arqueointensidad de los 6 especímenes obtenidos de una misma muestra (tiesto CM01). En la parte central de la figura se encuentran graficados los diagramas de Arai desplazados para resaltar su paralelismo y, por tanto, la similitud de arqueointensidades obtenidas.

Las arqueointensidades obtenidas del total de las muestras analizadas varían entre $(23.3 \pm 1.4) \mu \mathrm{T}$ y $(42.9 \pm 2.4) \mu \mathrm{T}$.

Los resultados del fechamiento arqueomagnético (intensidad) de tres muestras con edades previas (tiestos 7, 8 y 9) se presentan en la Figura 7. En cada caso se observan tres posibles intervalos, siendo el intervalo central el que coincide con las cronologías previas obtenidas por ${ }^{14} \mathrm{C}$ (Capdepont et al., 2016; Castiñeira et al., 2010; Gianotti, 2005; Sans, 1985) y por termoluminiscencia [TL (Capdepont, 2013)].
En la Tabla 3 se resumen los resultados obtenidos en este trabajo, incluyendo los intervalos de edad más probables para las muestras, incluyendo las dos muestras sin fechamiento por métodos convencionales. Los intervalos de edad más probables estimados corresponden a edades entre 1660 AEC y 1660 EC, las cuales son consistentes con los datos cronológicos de ${ }^{14} \mathrm{C}$ y TL reportados previamente.

\section{Discusión y comentarios finales}

Cuatro de las cinco muestras pertenecientes al departamento de Rocha muestran intensidades similares, entre los $38.1 \pm 7.7 \mu \mathrm{T}$ y $42.9 \mu \mathrm{T} \pm 2.4 \mu \mathrm{T}$, con una datación arqueomagnética de $\sim$ [1600AEG - 1250AEG]. Dos de éstas (CM01 y tiesto 5) presentan una correlación bastante buena con dataciones previas por ${ }^{14} \mathrm{C}$ reportadas en Castiñeira et al. (2010) y Capdepont
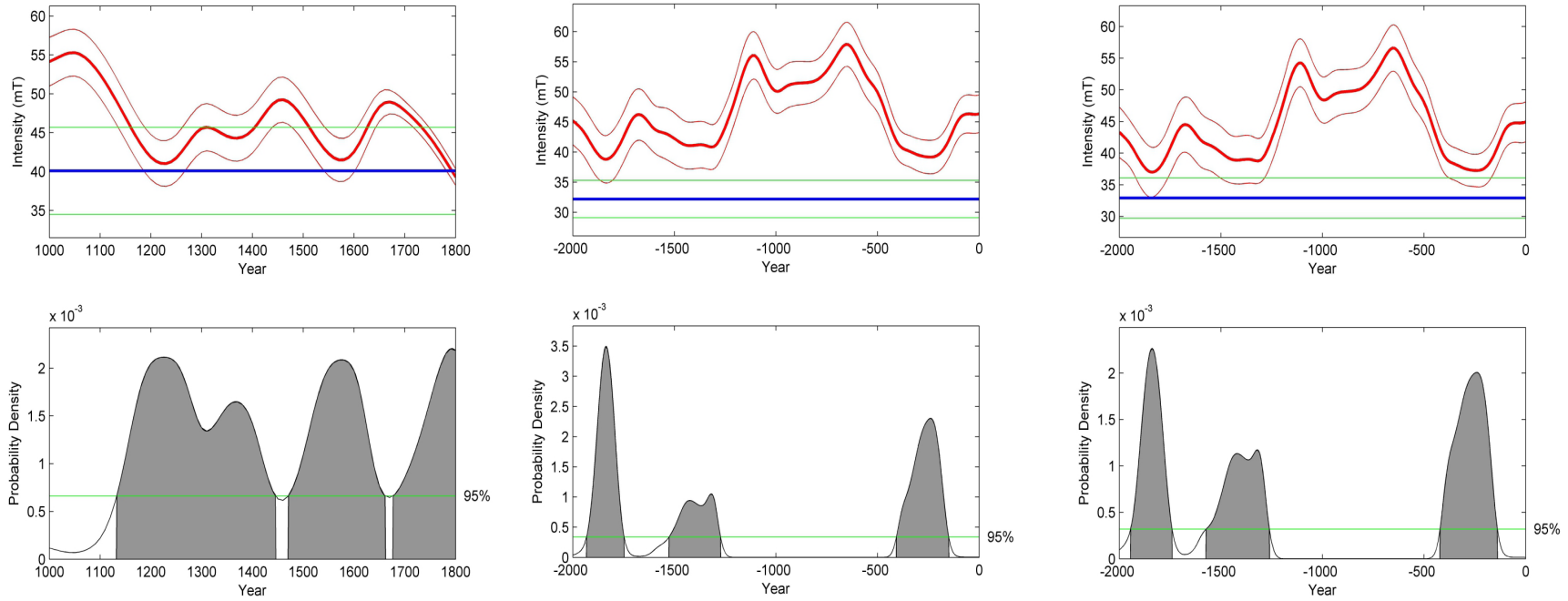

Tiesto 7 (Pasayndú)

Tiesto 8 (Rocha)

[1932AC 1748AC]

[1526AC 1273AC]

Tiesto 9 (Tacuarembó)

[1133DC 1445DC]

[407AC 150AC]

[1944AC 1741AC]

[1537AC 1262AC]

[421AC 140AC]

Figura 7 Fechamiento arqueomagnético (intensidad) de 3 muestras con fechamiento previo (tiestos 7,8 y 9). Paneles superiores: curvas de referencia de intensidad (curvas sólidas gruesas) con la envoltura al 95\% de error (curvas delgadas) calculadas a partir del modelo de campo geomagnético global SHA.DIF.14k (Pavón-Carrasco et al., 2014) y los datos sin fecha -arqueointensidad (líneas rectas gruesas) con la envoltura de error al 95\% (líneas rectas delgadas). Paneles inferiores: función de densidad de probabilidad (PDF), calculado a nivel de $95 \%$ de probabilidad, después de la comparación del valor medio de arqueointensidad del sitio con la curva de referencia SHA. DIF.14k (Pavón-Carrasco et al., 2014). 
et al. (2016). Sin embargo, las edades ${ }^{14} \mathrm{C}$ calibradas para las otras dos muestras (CM03 y tiesto CO13) son más tardías que sus correspondientes dataciones arqueomagnéticas. En este punto vale la pena recordar que se están datando materiales diferentes: carbón vegetal, por un lado, y la propia cerámica, por el otro lado, en el primero de los casos (muestra CM03). Para el segundo caso (tiesto CO13), se están datando materia orgánica en un sedimento y, nuevamente, la propia cerámica. La quinta de ellas (tiesto CO08) presenta, sin embargo, un valor $\sim 40 \%$ más bajo que las otras cuatro muestras, para el cual no fue posible estimar un intervalo de edad confiable.

Tres cerámicas provenientes del sitio Guayacas (Departamento de Paysandú) muestran intensidades similares, con una variación entre (40.1 \pm 5.6 y $46.3 \pm 12.9) \mu \mathrm{T}$. Cabe destacar que estas muestras son las únicas que pertenecen a momentos más tardíos (Capdepont, 2013), siendo las cronologías propuestas para las mismas [1471EG - 1656EG], las cuales corresponden bastante bien con las cronologías obtenidas por TL.

En general, las dataciones de las muestras restantes concuerdan con las dataciones por ${ }^{14} \mathrm{C}$ de las zonas correspondientes. A partir de los resultados obtenidos en este estudio, se proponen temporalidades para piezas carentes de cronologías previas.

Todas las muestras poseen edades correspondientes al Holoceno tardío inicial, a excepción de las localizadas en el sitio Guayacas que se acercan más al momento de contacto entre grupos indígenas y europeos.

Las cronologías presentadas en este trabajo son los primeros fechamientos arqueomagnéticos

Tabla 3. Fechamientos arqueomagneticos. Descripción de las diferentes columnas. Muestra: nombre del tiesto; Ubicación: zona de proveniencia de los tiestos; Departamento: Uruguay está formado por 19 departamentos, que son las entidades subnacionales de primer orden; Edad: fechamientos disponibles de la zona por diferentes métodos; método: de fechamiento (TLD: Termoluminiscencia; 14C: radio carbono; ND: no disponible); material: tipo de material utilizado para el fechamiento (COH: Capa de ocupación humana; CV: Carbón vegetal; C: Cerámica); Al: arqueointensidad absoluta determinada; 1 $\sigma$ : desviación estándar; Intervalo probable: El intervalo de edad estimado arqueomagnéticamente. Los renglones resaltados en Negrita corresponden a muestras con fechamientos disponibles por otros métodos.

\begin{tabular}{|c|c|c|c|c|c|c|c|c|}
\hline Muestra & Ubicación & Departamento & $\begin{array}{c}\text { Edad } \\
\text { aP }\end{array}$ & método & material & $\begin{array}{l}\mathrm{AI} \\
\mu \mathrm{T}\end{array}$ & $\begin{array}{c} \pm 1 \sigma \\
\mu \mathrm{T}\end{array}$ & $\begin{array}{c}\text { Intervalo } \\
\text { probable }\end{array}$ \\
\hline T47 & Yaguarí & Tacuarembó & $3060 \pm 45$ & ${ }^{14} \mathrm{C}$ & CV & 40.3 & 2 & $\begin{array}{l}\text { 1660AEC } \\
\text { 1229AEC }\end{array}$ \\
\hline Tiesto 7 & $\begin{array}{l}\text { Guayacas } \\
\text { Sector B4 }\end{array}$ & Paysandú & 400 & TLD & C & 40.1 & 5.6 & $\begin{array}{l}\text { 1471EC } \\
1661 \mathrm{EC}\end{array}$ \\
\hline Tiesto 8 & Costa lagunar & Rocha & $\sim 3050$ & $14 \mathrm{C}$ & $\mathrm{COH}$ & 32.2 & 3.1 & $\begin{array}{l}\text { 1526AEC } \\
\text { 1273AEC }\end{array}$ \\
\hline Tiesto 9 & Cerrito & Tacuarembó & $\sim 3000$ & $14 \mathrm{C}$ & CV & 32.9 & 3.2 & $\begin{array}{l}\text { 1537AEC } \\
\text { 1262AEC }\end{array}$ \\
\hline Tiesto 10 & Costa & Canelones & ND & & & 44.1 & 9.1 & \\
\hline Tiesto 11 & Duna & Rocha & ND & & & 38.2 & 3.9 & \\
\hline Tiesto 12 & Costa arroyo & Paysandú & ND & & & 34 & 0.8 & \\
\hline Tiesto 01 & $\begin{array}{l}\text { Cerrito Laguna de } \\
\text { Castillos }\end{array}$ & Rocha & ND & & & 42.9 & 2.4 & \\
\hline Tiesto 03 & $\begin{array}{l}\text { Cerrito Laguna de } \\
\text { Castillos }\end{array}$ & Rocha & ND & & & 42.7 & 5.9 & \\
\hline Tiesto 05 & La Esmeralda & Rocha & ND & & & 38.1 & 7.7 & \\
\hline Tiesto 29 & Yale 27 & Tacuarembó & ND & & & 26.4 & 1.7 & \\
\hline $\mathrm{CO} 08$ & García Ricci & Rocha & ND & & & 23.3 & 1.4 & \\
\hline $\mathrm{CO} 13$ & García Ricci & Rocha & ND & & & 39.2 & 4.4 & \\
\hline Gy0633 & Guayacas & Paysandú & ND & & & 42.6 & 4.7 & \\
\hline Gy04042 & Guayacas & Paysandú & ND & & & 46.3 & 12.9 & \\
\hline
\end{tabular}


de tiestos cerámicos elaborados por sociedades indígenas de Uruguay. Asimismo, el trabajo muestra la utilidad de esta metodología como medio alternativo de datación para obtener cronologías precisas relacionadas con la producción y dispersión de la cerámica indígena de Uruguay.

\section{Agradecimientos}

AG agradece el apoyo financiero de los proyectos CONACyT 252149 y UNAM-PAPIIT IN105214 y 101717 . Se agradece la participación de María de la Luz Gómez Rivera en el trabajo experimental.

\section{Referencias}

Austral, A., 1977, Arqueología de urgencia en el Yacimiento de Bañadero: Uruguay, Seminario sobre Medio Ambiente y Represas, 2, 3-20.

Bowles, J., Gee, J., Hildebrand, H.J., Tauxe, L., 2002, Archeomagnetic intensity results from California and Ecuador: evaluation of regional data: Earth and Planetary Science Letters, 203, 967-81.

Capdepont, I., 2013, Arqueología de sociedades indígenas del litoral del río Uruguay: Alemania, Publicia, 9-80.

Capdepont, I., Castiñeira, C., del Puerto, L., Fernández, G., 2016, Desarrollo de las ocupaciones humanas durante el Holoceno en la cuenca de la Laguna de Castillos (Uruguay): síntesis y actualización de las investigaciones arqueológicas: TESSITURAS, 4(1), 53-93.

Castiñeira, C., Panario, D., Bracco, R., Gutiérrez, O., 2010, Concheros en la costa atlántica uruguaya y su vinculación con la dinámica litoral, en Arqueología Argentina en los inicios de un Nuevo Siglo: Rosario, Laborde Libros Editor, 635-643.

Chauvin, A., Garcia, A., Lanos, Ph., Laubenheimer, F., 2000, Paleointensity of the geomagnetic field recovered on archaeomagnetic sites from
France: Physics of the Earth and Planetary Interiors, 120, 111-36.

Cobas, I., Prieto, M.P., 1998, Regularidades espaciales en la cultura material: la cerámica de la Edad de Bronce y la Edad de Hierro en Galicia: Gallaecia, 17, 151-175.

Coe, R.S., 1967, Paleointensities of the Earth's magnetic field determined from Tertiary and Quaternary rocks: Journal of Geophysical Research, 72, 3247-3262.

Coe, R., Grommé, S., Mankinen, E.A., 1978, Geomagnetic paleointensities from radiocarbon-dated lava flows on Hawaii and the question of the Pacific non-dipole low: Journal of Geophysical Research, 83, 1740-1756.

Díaz, A., M. Fornaro, 1977, Intento de sistematización de las Modalidades Alfareras del Litoral Uruguayo, en V Encuentro de Arqueología del Litoral: Ministerio de Educación y Cultura e Intendencia Municipal de Río Negro, 165-174.

Gianotti, C., 2005, Intervenciones arqueológicas en el cerrito 27 del conjunto Lemos: Revista TAPA, 36, 79-98.

Goguitchaichvili, A., Loponte, D., Morales, J., Acosta, A., 2012, The Archaeointensity of the Earth's Magnetic Field retrieved from Pampean Ceramics (South America): Archaeometry, 54 (2), 388-400.

Hartmann, G.A., Trindade, R.I.F., Goguitchaichvili, A., Etchevarne, C., Morales, J., Afonso, M.C., 2009, First archeointensity results from Portuguese potteries (1550-1750 AD): Earth Planets Space, 61, 93-100.

Hilbert, K. 1991, Aspectos de la Arqueología en Uruguay: Alemania, Mainz am Rhein Zabern, $52 \mathrm{p}$.

Leonhardt, R., 2006, Analyzing rock magnetic measurements: The Rock-MagAnalyzer 1.0 software: Computers \& Geosciences, 32, 1420-1431.

Leonhardt, R., Heunemann, C. Krása, D., 2004, Analyzing absolute paleointensity determinations: acceptance criteria and the 
software ThellierTool4.0, Geochemistry, Geophysics, Geosystems, 5(12), Q12016.

McCabe, G., Jackson, M., Ellwood, B., 1985, Magnetic anisotropy in the Trenton limestone: results of a new technique, anisotropy of anhysteric susceptibility: Geophysical Research Letters, 12, 333-6.

Morales, J., Goguitchaichvili, A., UrrutiaFucugauchi, J., 2007, Cooling rate effect as a cause of systematic overestimating of the absolute Thellier paleointensities: A cautionary note: Studia Geophysica et Geodaetica, 51, 315-326.

Morales, J., Goguitchaichvili, A., Acosta, G., González-Morán, T., Alva-Valdivia, L., Robles-Camacho, J., HernándezBernal, M.S., 2009, Magnetic properties and archeointensity determination on Pre-Columbian pottery from Chiapas, Mesoamerica: Earth Planets \& Space, 61, 83-91.

Morales, J., Goguitchaichvili, A., Aguilar-Reyes, B., Pineda-Duran, M., Camps, P., Carvallo, C. Calvo-Rathert, M., 2011, Are ceramics and bricks reliable absolute geomagnetic intensity carriers?: Physics of the Earth and Planetary Interiors, 187, 310-321.

Morales, J., Goguitchaichvili, A., Aguilar, B., Pineda, M., Carvallo, G., Baramendi, L., Hernandez, G., Oliveros A., 2012, A detailed rock-magnetic and archeointensity investigation on some pottery relics and burned floors from Tzintzuntzan Archeological Site, Western Mesoamerica: Geoarchaeology, 27 (6), 521-537.

Pavón-Carrasco, F. J., Rodriguez-Gonzalez, J., Osete, M. L., Torta, J., 2011, A Matlab tool for archaeomagnetic dating: Journal of Archaeological Science, 38 (2), 408-419.

Pavon-Carrasco, FJ., Osete, M.L., Torta, J.M., De Santis, A., 2014, A geomagnetic field model for the Holocene based on archaeomagnetic and lava flow data: Earth and Planetary Science Letters, 388, 98-109.
Prieto, M.P., 2003, La cerámica neolítica en Galicia. Estudio de la síntesis desde la perspectiva de la Arqueología del Paisaje, en Actas del III Congreso del Neolítico de la Península Ibérica: Pablo Arias Cabal, Roberto Otañón Peredo, Gristina García-Moncó Piñeiro (eds.), Universidad de Cantabria, 337-348.

Rice, P.M. 1987, Pottery analysis: a sourcebook: Chicago, University of Chicago Press, 561 p.

Riisager, P., Riisager, J., 2001, Detecting multidomain magnetic grains in Thellier palaeointensity experiments: Physics of the Earth and Planetary Interiors, 125, 111-117.

Sans, M., 1985, Arqueología de la región de Yaguarí, Depto. De Tacuarembó, en Estado de las investigaciones arqueológicas en el Uruguay: Montevideo, CEA Publicaciones (3), 57-61.

Selkin, P. A., Gee, J. S., Tauxe, L., Meurer, W. P., Newell, A. J., 2000, The effect of remanence anisotropy on paleointensity estimates: a case study from the Archean Stillwater Complex: Earth and Planetary Science Letters, 183, 403-16.

Serrano, A., 1933, Las culturas protohistóricas del Este Argentino y Uruguay: Memorias del Museo de Paraná, 7, 9-43.

Serrano, A., 1936, Etnografia de la antigua provincia del Uruguay: Argentina, Talleres Gráficos Paraná, 207 p.

Serrano, A., 1972, Líneas fundamentales de la Arqueología del Litoral (una tentativa de periodización):Córdoba, Publicaciones de la Universidad Nacional de Córdoba, Instituto de Antropología XXXII, 79 p.

Shaw, J., Walton, D., Yang, S., Rolph, T.C., Share, J.A., 1996, Microwave archaeointensities from Peruvian ceramics: Geophysical Journal International, 124, 241-4.

Shepard, A., 1954, Ceramics for the archaeologist: Washington, D.C, Carnegie Institution of Washington, Publication 609, 447 p.

Thellier, E., Thellier, O., 1959, Sur l'intensité du champ magnétique terrestre dans le passé historique et géologique: Annals of Geophysics, 15, 285-376. 